\title{
Classical and quantum dispersion In Robertson-Walker cosmologies
}

\author{
Roman Tomaschitz \\ Dipartimento di Matematica Pura ed Applicata dell' Università degli Studi di Padova, \\ Via Belzoni 7, I-35131 Padova, Italy, and Theoretical Physics Group, Tata \\ Institute of Fundamental Research, Homi Bhabha Road, Bombay 400 005, India
}

(Received 11 June 1992; accepted for publication 15 October 1992)

\begin{abstract}
The instability of world lines in Robertson-Walker universes of negative spatial curvature is investigated. A probabilistic description of this instability, similar to the Liouville equation, is developed, but in a manifestly covariant, nonHamiltonian form. To achieve this the concept of a horospherical geodesic flow of expanding bundles of parallel world lines is introduced. An invariant measure and a covariant evolution equation for the probability density on which this flow acts is constructed. The orthogonal surfaces to these bundles of trajectories are horospheres, closed surfaces in three-space, touching the boundary at infinity of hyperbolic space, where the flow lines emerge. These horospheres are just the wave fronts of spherical waves, which constitute a complete set of eigenfunctions of the Klein-Gordon equation. This fact suggests that the evolution of the quantum mechanical density with the classical one be compared, and asymptotic identity in the asymptotically flat region is found. This leads, furthermore, to the study of the time behavior of the dispersion of the energy and the coordinates and the energy-time uncertainty relation, and identity in the late stage of the cosmic evolution is again found. In an example it is finally demonstrated that this identity can persist in the early phase of the expansion with a rapidly varying scale factor, provided the fields are conformally coupled to the curvature.
\end{abstract}

\section{INTRODUCTION}

One of the most remarkable features of Robertson-Walker (RW) cosmologies of negative spatial curvature is the instability of the classical geodesic trajectories, the probabilistic character of world lines. This instability, foreign to both the closed models and the models with Euclidean spacelike sections, does not seem to have gained the attention in the literature that it deserves.

The most efficient and quantitative way to describe such systems that are highly sensitive with respect to the choice of the initial conditions is that of statistical mechanics, in terms of classical probability densities and covariant evolution equations. We study the dispersion of these densities in terms of classical energy-time uncertainty relations, designed after the conventional quantum mechanical ones. We do this in quite a general context, for arbitrary expansion factors, however, we restrict ourselves in this paper to topologically simply connected cosmologies, and take as the spacelike slices the Minkowski hyperboloid (mass shell). The formalism that we adopt is nevertheless designed in a way that it is generalizable to cosmologies whose spacelike slices are arbitrary hyperbolic manifolds. ${ }^{1-3}$ In such cosmologies exact relations between bound-state wave fields and chaotic trajectories have been derived, and it is clear that the next question we have to pose is whether there persist such relations for wave fields of the continuous spectrum and the remaining unstable but not chaotic trajectories. In the case of the trivial topology of the mass shell, neither bound states nor chaotic trajectories exist, we only have to deal with unstable world lines and the continuous spectrum of the wave equation.

We shall study the Klein-Gordon evolution of wave packets, their densities and currents, 
and compare them with the evolution of the classical horospherical flow, its density, and current. We obtain, with a suitable choice of the initial conditions, the asymptotic identity between the classical and quantum evolution of the mentioned quantities. Likewise, we get this identity for the wave mechanical and the classical $\Delta E \Delta t$, which shows that the instability of the classical world lines can produce, even quantitatively, the same dispersion phenomena as quantum mechanics. We demonstrate this in two examples, studying the time evolution of $\Delta E \Delta t$ during the initial and final stage of the cosmic expansion.

The paper is organized as follows. In Sec. II we introduce the concept of a horospherical flow. That needs some introductory comments. Usually a nonrelativistic geodesic flow is treated in a Hamiltonian context, as an initial value problem, by specifying the initial coordinates and momenta. Now, because of the Lyapunov instability of the flow lines with respect to a variation of the initial conditions one has in practice to pass over to the Liouville equation, and to study the evolution of the probability density. Our relativistic approach is guided by quantum mechanics. Let us consider a spherical wave, generated at some point at infinity of hyperbolic space. Its wave fronts are horospherical, ${ }^{4,5}$ namely, closed surfaces of constant positive curvature, tangent to the boundary at infinity of hyperbolic space at some point, say $\boldsymbol{\eta}$. The orthogonals to these horospheres are just the geodesics issuing from $\eta$. These expanding bundles of parallel geodesics constitute our horospherical flow. Having chosen a point $\boldsymbol{\eta}$ from which the bundle emerges, a flow line is, in principle, modulo its instability, determined by choosing a point of space-time through which it passes, and the energy at this point. We give the explicit construction of this horospherical flow and its action, which is closely related to the space part of the wave fields of the Klein-Gordon equation.

In Sec. III we construct the invariant measure of the horospherical flow, and the covariant evolution equation of the classical density. Instead of the initial distribution of the momentum, we have now to specify the initial spread of the energy and the width of the cone (=spread of the $\eta$ values) from which the flow lines emerge. In Sec. IV we compare the quantum evolution according to the Klein-Gordon equation with the evolution of the classical density under the horospherical flow, and proof asymptotic equivalence in a period of slow variation of the expansion factor. The expansion in the late stage of the cosmic evolution is very likely to be adiabatic, if the curvature of three-space is negative. In Sec. V we study the dispersion of classical and quantum densities. In particular, we calculate the time evolution of the product $\Delta E \Delta x$, the dispersion of the energy and the coordinates. As is not surprising after Sec. IV, we get equivalence of the classical and quantum evolution for $t \rightarrow \infty$, but we also show in an example that this equivalence can persist for $t \rightarrow 0$ and rapidly varying expansion factors. In Sec. VI, finally, we come to our conclusions and discuss the foregoing a little with respect to RW cosmologies whose spacelike slices are multiply connected hyperbolic manifolds.

We start by summarizing some basic formulas. ${ }^{1-3}$ The scalar wave fields we consider satisfy the Klein-Gordon equation

$$
\left[\square-\xi \hat{R}-(m c / \hbar)^{2}\right] \psi=0,
$$

where $\square$ is the Laplace-Beltrami operator of the RW-line element,

$$
d \sigma^{2}=-c^{2} d t^{2}+a^{2}(t) d s^{2}
$$

in $\mathbb{R} \times B^{3}$ or $\mathbb{R} \times H^{3}$ (see the Appendix, where we also summarize our basic notation). $d s^{2}$ is the line element of hyperbolic three-space, $a(t)$ the expansion factor, $\xi$ the coupling to the curvature scalar $\hat{R}$ of (1.2).

If we make the separation ansatz,

$$
\psi=\varphi(t) P^{1-i s}(\mathbf{x}, \boldsymbol{\eta})
$$


with the Poisson kernel $P$ as in Eqs. (A2) or (A9), we arrive at Eq. (A3) for the space part, and obtain

$$
\ddot{\varphi}+3 \frac{\dot{a}(t)}{a(t)} \dot{\varphi}+\left[\left(m c^{2} / \hbar\right)^{2}+\Lambda^{2}\left(1+s^{2}\right) a^{-2}(t)+c^{2} \xi \hat{R}(t)\right] \varphi=0
$$

for the time dependence. We normalize the solutions by imposing

$$
\frac{1}{2}(\dot{\varphi} \varphi-\dot{\varphi} \bar{\varphi})= \pm i a^{-3}(t)
$$

We want to study the dispersion of the density,

$$
\rho=\frac{1}{2 i}\left(\Psi \frac{\partial}{\partial t} \bar{\Psi}-\bar{\Psi} \frac{\partial}{\partial t} \Psi\right)
$$

constructed of wave packets,

$$
\Psi(\mathbf{y}, t)=\frac{1}{\sqrt{2 \pi \alpha}} \frac{1}{2 \pi \gamma^{2}} \int_{\mathbf{R}^{3}} s \varphi(s, t) P^{1-i s}(\mathbf{y}, t ; \xi) \exp \left[\frac{-\left(s-s_{0}\right)^{2}}{2 \alpha^{2}}-\frac{\left(\xi-\xi_{0}\right)^{2}}{2 \gamma^{2}}\right] d \xi d s .
$$

In Refs. 2 and 3, the following formula for the energy of the wave fields (1.3) has been derived:

$$
\begin{aligned}
\epsilon(s, t)= & \frac{1}{2} \hbar a^{3}\left\{\left(\varphi_{, t}+6 \xi \varphi \frac{\dot{a}}{a}\right) \overline{\left(\varphi_{, t}+6 \xi \varphi \frac{\dot{a}}{a}\right)}+\varphi \bar{\varphi}\left[\left(\frac{m c^{2}}{\hbar}\right)^{2}\right.\right. \\
& \left.\left.+\Lambda^{2}\left[s^{2}+1-6 \xi\right] a^{-2}+6 \xi(1-6 \xi) \frac{\dot{a}^{2}}{a^{2}}\right]\right\}
\end{aligned}
$$

$\varphi$ subject to (1.4) and (1.5).

Using the general formula ${ }^{3}$ for the energy-momentum tensor for solutions of (1.1), and the orthogonality relations (A4), one readily obtains

$$
E(\Psi, t)=\frac{1}{\sqrt{\pi} \alpha} \int_{-\infty}^{+\infty} \epsilon(s, t) \exp \left[\frac{-\left(s-s_{0}\right)^{2}}{\alpha^{2}}\right] d s
$$

for the energy of the wave packets (1.7).

\section{THE CLASSICAL HOROSPHERICAL FLOW AND ITS BOUNDARY ACTION}

The simplest way to calculate the classical flow and its action $S$ is to map geodesics in $B^{d+1}$ (which are circular arcs orthogonal to the boundary sphere $S_{d}$ ) onto straight lines through the origin and to solve then the one-dimensional problem. The mapping can be performed by Möbius transformations, leaving the hyperbolic line-element invariant; see the Appendix.

The reduced, i.e., one-dimensional Lagrangian reads as

$$
L_{\mathrm{red}}^{2}=c^{2}\left(\frac{d t}{d s}\right)^{2}-\frac{4 a^{2}}{\left(1-r^{2} / R^{2}\right)^{2}}\left(\frac{d r}{d s}\right)^{2} \equiv \lambda>0,
$$

with the solutions 
pp. 1025-36 in attached file 
discussed on completely equal footing; however, there are several special cases to consider, and that will be communicated elsewhere.

\section{CONCLUSION AND OUTLOOK}

We are continuing our work on the dynamics of fundamental particles in open and expanding RW cosmologies of negative spatial curvature. In these cosmologies there do exist common features between the deterministic but unstable classical mechanics and its quantized counterpart, ${ }^{1,9}$ which are usually not encountered in other dynamical systems.

Due to the instability of the world lines one needs, even in the classical description, probability densities. Expanding bundles of geodesic flow lines act on them and generate dispersion. These bundles constitute just the orthogonal trajectories for the wave fronts of the horospherical waves, emanating from a point at infinity of hyperbolic space, and these waves constitute, in turn, a complete set of eigenfunctions for the Klein-Gordon equation. Therefore we could show (Sec. V) the equivalence of the classical and quantum dispersion of the energy and the coordinates in the asymptotically flat region, in periods of adiabatic expansion, and also in special cases at the beginning of the cosmic expansion.

Let us now shortly point out how the foregoing can be generalized to RW cosmologies with open hyperbolic manifolds as spacelike sections. We break at this point with self-containedness, a certain familiarity with Refs. 1, 3, and 9 is useful for the understanding of the following suggestions. At first some comments on these cosmologies. They are determined by the choice of the expansion factor, the topology of three-space, and the metric of three-space, which may itself be time dependent, since a hyperbolic three-manifold if it is open and multiply connected can carry many nonisometric metrics of constant curvature $-1 / R^{2}$. In fact, the space of these metrics can be parametrized by a certain number $n$ of real parameters, varying in a finite domain of $R^{n}$ (deformation space), $n$ depending on the topology. During the cosmic evolution the metric of three-space is determined by a time-dependent path in the deformation space.,9

Let us, at first, discuss the choice of the expansion factor. If we discard periodic universes and de Sitter space, Einstein's equations and negative curvature require essentially linear behavior of the expansion factor in the final stage of the expansion. In the intermediate stage one cannot say much about it; there may be periods of rapid variation and even oscillation alternating with phases of slow expansion, and cosmological considerations should not depend on the knowledge of $a(t)$ in this region. Finally, in the early stage we can make guesses about the asymptotic decay of $a(t)$; for example, if we assume power law behavior, $a(t) \sim t^{\lambda}$, we get qualitatively different behavior of the classical and quantum dynamics in only four $\lambda$ intervals, ${ }^{2}$ namely, $\left(0, \frac{1}{3}\right),\left(\frac{1}{3}, \frac{2}{3}\right),\left(\frac{2}{3}, 1\right)$, and $(1, \infty)$.

Next, we make some comments on the topology of three-space. Homogeneity and isotropy require constant curvature, but let the topology be open. It seems to be rather unjustified to appeal to the three-sphere for reasons of simplicity, and to the closure of three-space because of Mach's principle, which, attractive as it may be, has never been able to leave the realm of philosophy.

Which three-manifolds of constant curvature come in question as possible candidates for three-space? Three-manifolds of positive and zero curvature are very exceptional. ${ }^{10}$ Then, there are the hyperbolic manifolds of finite volume, typical examples for them are the platonic solids with face identification, and they are also rather artificial. Thus we are left over with the open hyperbolic manifolds. Their classification is not yet completed, however, there seem to emerge, apart from an enormous amount of more or less pathological counter examples, two generic classes: the massive handlebodies, topologically the product of a finite interval and a disk with some smaller discs removed, and the thickened surfaces, products of a finite interval and a Riemann surface. Now, what is the connectivity of three-space, and in the second case what is the genus of its fibers? In my opinion there are only two cases to distinguish, either it is high, or it is low, only that will make a qualitative difference concerning the dynamics. With respect 
to the possible choices of the metric of three-space, which is, as mentioned, not uniquely determined by the topology, my answer is similar. Either the metric is well in the interior of the deformation space, or close to the boundary, only that will make a qualitative difference, and, of course, the time variation of the deformation path.

Finally it is rather straightforward to generalize the results of this paper to these cosmologies. The wave equation has already been discussed in this context, ${ }^{1}$ namely on fundamental polyhedra in the Poincaré ball representing the hyperbolic manifold. Analogously, the classical evolution equations (3.11) and (3.14) are adaptable by periodization with respect to the discrete group $\Gamma$, generated by the face-identifying Möbius transformations of the fundamental polyhedron. Horospheres can be projected like geodesics into the manifold, for they have constant curvature. ${ }^{4}$ There are two cases to distinguish horospheres that emanate from the limit set $\Lambda(\Gamma)$ of $\Gamma$ and horospheres that emanate from its complement. The second case is similar to that treated in this paper, the projections remain closed, in general self-intersecting surfaces tangent at some point to the boundary at infinity of the three-manifold. In the first case, however, these projections constitute the surfaces of constant action of the chaotic trajectories, as well as the wave fronts of the chaotic wave fields, and their topology can get quite intricate. This and the topological scattering effects that arise are discussed in a subsequent paper. $^{11}$

\section{ACKNOWLEDGMENT}

The author acknowledges the support of the European Communities through their Science Programme under Grant No. B/SC1*-915078.

\section{APPENDIX: THE ORTHOGONALITY AND COMPLETENESS RELATIONS FOR THE LAPLACE OPERATOR IN $\boldsymbol{H}^{d+1}$ AND $B^{d+1}$, AND THE INVARIANCE GROUP OF HYPERBOLIC SPACE}

In this appendix we collect some basic formulas of hyperbolic spectral geometry and sketch the action of the Lorentz group in hyperbolic space, represented as Möbius transformations in the Poincare ball. To perform calculations in hyperbolic space it is very useful, in fact indispensable, to switch quickly from the ball model to the half-space model and vice versa, exploiting the spherical symmetry of $B^{d+1}$ and the cylindrical of $H^{d+1}$ in problems, where there is a special direction distinguished, e.g., by choosing a special point on the boundary. We also let the dimension $d+1, d>1$ of hyperbolic space open.

\section{The $H^{d+1}$ model} metric

$H^{d+1}$ is the half-space $\mathbb{R}^{d} \times \mathbb{R}^{+}$, parametrized by $(\mathbf{y}, t), \mathbf{y} \in \mathbb{R}^{d}, t \in \mathbb{R}$, and endowed with the

$$
d s^{2}=R^{2} t^{-2}\left(d t^{2}+d \mathbf{y}^{2}\right),
$$

and sectional curvature $-1 / R^{2}$. The volume element we denote by $d y_{H^{d+1}}$ and the LaplaceBeltrami operator by $\Delta_{H^{d+1}}$. The generalized eigenfunctions are powers of the Poisson kernel,

$$
P(\mathbf{y}, t ; \xi)=\frac{R t}{|\mathbf{y}-\xi|^{2}+t^{2}}, \quad \xi \in \mathbb{R}^{d},
$$

satisfying ${ }^{12,13}$

$$
-\Delta_{H^{d+1}} P^{\alpha}(\mathbf{y}, t ; \xi)=R^{-2} \alpha(d-\alpha) P^{\alpha}(\mathbf{y}, t ; \xi),
$$


$\alpha$ may be any complex number. We define the spectral variable $\lambda=\alpha(d-\alpha), \alpha=d / 2-i s, s \in \mathbb{R}$, the absolutely continuous spectrum lies in $\left[d^{2} / 4, \infty\right]$. The $\xi$ in (A2) is a degeneration index, also $\pm s \leftrightarrow \lambda$. There are no bound states. We have the following orthogonality relation:

$$
\begin{gathered}
\lim _{\epsilon \rightarrow 0} \int_{H^{d+1}} P^{d / 2-i s_{1}}\left(\mathbf{y}, t ; \xi_{1}\right) P^{d / 2+i s_{2}}\left(\mathbf{y}, t ; \xi_{2}\right)\left(\frac{t}{R}\right)^{\epsilon} d y_{H^{d+1}} \\
\quad=2 \pi^{d+1} \frac{\left|\Gamma\left(i s_{1}\right)\right|^{2} R^{2 d+1}}{\left|\Gamma\left(d / 2+i s_{1}\right)\right|^{2}} \delta\left(\xi_{1}-\xi_{2}\right) \delta\left(s_{1}-s_{2}\right) .
\end{gathered}
$$

In the case $d=2$ the right-hand side is thus $2 \pi^{3} R^{5} s_{1}^{-2} \delta\left(\xi_{1}-\xi_{2}\right) \delta\left(s_{1}-s_{2}\right)$. The integral in (A4) is standard; ${ }^{13}$ for in the $H^{d+1}$ model the Poisson kernel takes the form of a Feynman propagator. The completeness relation reads as

$$
\lim _{\epsilon \rightarrow 0} \int_{\mathbf{R}^{d+1}} d \sigma_{H^{d+1}}(\xi, s) e^{-\epsilon|s|} P^{d / 2-i s}\left(\mathbf{y}_{1}, t_{1} ; \xi\right) P^{d / 2+i s}\left(\mathbf{y}_{2}, t_{2} ; \xi\right)=\delta_{H^{d+1}}\left(\mathbf{y}_{1}, t_{1} ; \mathbf{y}_{2}, t_{2}\right)
$$

with the spectral measure

$$
d \sigma_{H^{d+1}}(\xi, s)=\frac{1}{4 \pi^{d+1} R^{2 d+1}} \frac{|\Gamma(d / 2+i s)|^{2}}{|\Gamma(i s)|^{2}} d \xi d s .
$$

A possible representation of the $H^{d+1}-\delta$ function is

$$
\delta_{H^{d+1}}=\lim _{\epsilon \rightarrow 0} \frac{\Gamma(1+d / 2)}{\pi^{d / 2+1} R^{d+1}} \frac{\epsilon}{\left(\epsilon^{2}+4 L\right)^{d / 2+1}},
$$

with the point pair invariant $L\left(\mathbf{y}_{1}, t_{1} ; \mathbf{y}_{2}, t_{2}\right)$ defined in (A16). The validity of (A7) can readily be seen by integrating it against a test function, using the symmetry (A17). The integral in (A5) is again a Feynman convolution with respect to $\xi$.

\section{The $B^{d+1}$ model}

$B^{d+1}$ is the ball $|\mathbf{x}|<R, \mathbf{x} \in \mathbb{R}^{d+1}$, endowed with the metric

$$
d s^{2}=\frac{4 d \mathbf{x}^{2}}{\left(1-|\mathbf{x}|^{2} / R^{2}\right)^{2}}
$$

the volume element $d y_{B^{d+1}}$, and the Laplacian $\Delta_{B^{d+1}}$. The Poisson kernel now reads ${ }^{12}$ as

$$
P(\mathbf{x}, \eta)=R^{2} \frac{1-|\mathbf{x}|^{2} / R^{2}}{|\mathbf{x}-\eta|^{2}}, \quad \eta \in S_{d},
$$

$S_{d}$ is the boundary sphere of $B^{d+1}$. Equation (A3) holds true with the obvious replacements.

The isometry $H^{d+1} \leftrightarrow B^{d+1}$ may be realized as ${ }^{5}$

$$
(\mathrm{y}, t) \rightarrow \frac{1}{\mathrm{y}^{2} / R^{2}+(1+t / R)^{2}}\left(2 \mathrm{y}, R\left(\frac{\mathrm{y}^{2}}{R^{2}}+\frac{t^{2}}{R^{2}}-1\right)\right),
$$

with the inverse

$$
\left(\mathbf{x}, x_{d+1}\right) \rightarrow \frac{R}{\mathbf{x}^{2} / R^{2}+\left(1-x_{d+1} / R\right)^{2}}\left(\frac{2 \mathrm{x}}{R}, 1-\frac{\mathbf{x}^{2}}{R^{2}}-\frac{x_{d+1}^{2}}{R^{2}}\right) .
$$


Note that $(0, R) \in H^{d+1}$ is mapped into the center of $B^{d+1}$, and the point at infinity on the boundary of $H^{d+1}$ is mapped into $(0, R) \in S_{d}$. By this transformation the boundary sphere $S_{d}$ of $B^{d+1}$ is stereographically projected onto the hyperplane $\mathbb{R}^{d}$ at infinity of $H^{d+1}$, and the spherical volume element on $S_{d}$ projects as

$$
d \Omega_{S_{d} \rightarrow 2^{d}} \frac{d \xi}{\left(1+|\xi|^{2} / R^{2}\right)^{d}}
$$

onto $\mathbb{R}^{d}$. With (A10), we have

$$
P(\mathbf{x}, \boldsymbol{\eta})=P(\mathbf{y}, t ; \xi)\left(1+|\xi|^{2} / R^{2}\right),
$$

where the boundary points $\eta \in S_{d}, \xi \in \mathbb{R}^{d}$ also correspond via (A10).

The orthogonality relation in $B^{d+1}$ reads as

$$
\int_{B^{d+1}} P^{d / 2-i s_{1}}\left(\mathbf{x}, \eta_{1}\right) P^{d / 2+i s_{2}}\left(\mathbf{x}, \eta_{2}\right) d y_{B^{d+1}}=(2 \pi)^{d+1} \frac{\left|\Gamma\left(i s_{1}\right)\right|^{2} R^{2 d+1}}{\left|\Gamma\left(d / 2+i s_{1}\right)\right|^{2}} \delta_{S_{d}}\left(\eta_{1}, \eta_{2}\right) \delta\left(s_{1}-s_{2}\right),
$$

where $\delta_{S_{d}}$ is the $\delta$ function on $S_{d}$. As a regularization of the integral one may take the factor $\exp -\epsilon L\left(\mathbf{x}, \mathbf{x}_{0}\right), L$ as in $(\mathrm{A} 16), \mathbf{x}_{0} \in B^{d+1}$. The $B^{d+1}$-completeness relation reads as

$$
\int_{S_{d} \times \mathbf{R}} d \sigma_{B^{d+1}}(\boldsymbol{\eta}, s) P^{d / 2-i s}\left(\mathbf{x}_{1}, \boldsymbol{\eta}\right) P^{d / 2+i s}\left(\mathbf{x}_{2}, \boldsymbol{\eta}\right)=\delta_{B^{d+1}}\left(\mathbf{x}_{1}, \mathbf{x}_{2}\right),
$$

with the spectral measure

$$
d \sigma_{B^{d+1}}(\eta, s)=\frac{1}{2(2 \pi)^{d+1} R^{2 d+1}} \frac{|\Gamma(d / 2+i s)|^{2}}{|\Gamma(i s)|^{2}} d \Omega_{S_{d}} d s .
$$

The same regularization for the integral as in (A5) may be used. The volume element of the $d$ sphere is not normalized. $\delta_{B^{d+1}}$ is the $\delta$ function of $B^{d+1}$. Equation (A7) holds true, with $\delta_{H^{d+1}}$ replaced by $\delta_{B^{d+1}}$, and $L$ in (A7) is the following point-pair invariant of hyperbolic space:

$$
L\left(\mathbf{x}_{1}, \mathbf{x}_{2}\right):=\frac{1}{R^{2}} \frac{\left|\mathbf{x}_{1}-\mathbf{x}_{2}\right|^{2}}{\left(1-\left|\mathbf{x}_{1}\right|^{2} / R^{2}\right)\left(1-\left|\mathbf{x}_{2}\right|^{2} / R^{2}\right)}=\frac{\left|\mathbf{y}_{1}-\mathbf{y}_{2}\right|^{2}+\left(t_{1}-t_{2}\right)^{2}}{4 t_{1} t_{2}}=: L\left(\mathbf{y}_{1}, t_{1} ; \mathbf{y}_{2}, t_{2}\right),
$$

and $\mathbf{x}_{1} \leftrightarrow\left(y_{1}, t_{1}\right), \mathbf{x}_{2} \leftrightarrow\left(y_{2}, t_{2}\right)$ correspond via (A10). $L$ is invariant with respect to the invariance group of hyperbolic space, the group of Möbius transformations $(\gamma)$ acting on $B^{d+1}$ or $H^{d+1}$, respectively [see (A21)-(A30)]:

$$
L\left(\gamma \mathbf{x}_{1}, \gamma \mathbf{x}_{2}\right)=L\left(\mathbf{x}_{1}, \mathbf{x}_{2}\right),
$$

and analogously in $H^{d+1}$.

The boundary of $H^{d+1}$ is just $\mathbf{R}^{d} \cup\{\infty\} \approx S_{d}$. By (A12) the Poisson kernel at $\xi=\infty$ may be chosen as

$$
P(t)=t / R \text {. }
$$

We have orthogonality, 


$$
\int_{0}^{\infty} P^{d / 2-i s}(t) P^{d / 2+i s_{0}}(t) R^{d} t^{-d-1} d t=2 \pi \delta\left(s-s_{0}\right)
$$

and completeness,

$$
\int_{-\infty}^{\infty} P^{d / 2-i s}(t) P^{d / 2+i s}\left(t_{0}\right) d s=2 \pi\left(\frac{t}{R}\right)^{d+1} R \delta\left(t-t_{0}\right),
$$

on the $t$ coordinate, i.e., in $L^{2}\left([0, \infty], t^{-d-1} d t\right)$.

To treat the horospherical flow in Sec. II we need some formulas for Möbius transformations. ${ }^{12}$ Here using the ball model and its spherical symmetry is almost compulsory. For $\mathbf{x}, \mathbf{y} \in B^{d+1}, \mathbf{x}=\left(x^{i}\right)$, we define

$$
\mathbf{x}^{*}:=\frac{R^{2} \mathbf{x}}{|\mathbf{x}|^{2}}, \quad Q_{i j}(\mathbf{x}):=\delta_{i j}-2 \frac{x^{i} x^{j}}{|\mathbf{x}|^{2}}
$$

and

$$
[\mathbf{x}, \mathbf{y}]:=\sqrt{1+|\mathbf{x}|^{2}|\mathbf{y}|^{2} / R^{4}-2 \mathrm{xy} / R^{2}}=R^{-2}|\mathbf{x}|\left|\mathbf{y}-\mathbf{x}^{*}\right|=R^{-2}|\mathbf{y}|\left|\mathbf{x}-\mathbf{y}^{*}\right| .
$$

There is a Möbius transformation, $T_{\mathrm{y}}(\cdot)$, acting in $B^{d+1}$ that maps $\mathrm{y}$ into the center $\mathbf{0}$ and leaves the straight line through $\mathbf{y}$ and $\mathbf{0}$ fixed. This transformation is unique up to a rotation around this straight line, and it can be realized as

$$
\mathrm{x} \rightarrow T_{\mathrm{y}} \mathbf{x}=\frac{\left(1-|\mathrm{y}|^{2} R^{2}\right)(\mathrm{x}-\mathrm{y})-|\mathbf{x}-\mathbf{y}|^{2} \mathbf{y} / R^{2}}{[\mathrm{x}, \mathbf{y}]^{2}} .
$$

This action extends to $S_{d}$. For $d=2$, using the stereographic projection (A10), $T_{\mathbf{y}} \mathbf{x}$ is just a Möbius transformation acting on the complex plane. The general form of a Möbius transformation in $B^{d+1}$ is (A23) followed by a rotation around the center $\mathbf{0}$. For the absolute value of (A23), we have

$$
\left|T_{\mathbf{y}} \mathbf{x}\right|^{2}=\frac{|\mathbf{x}-\mathbf{y}|^{2}}{[\mathbf{x}, \mathbf{y}]^{2}}=\frac{L(\mathbf{x}, \mathbf{y})}{1+L(\mathbf{x}, \mathbf{y})}
$$

with $L$ as in (A16). Thus

$$
\left|T_{\gamma \mathbf{y}} \gamma \mathbf{x}\right|=\left|T_{\mathbf{y}} \mathbf{x}\right|
$$

for every Möbius transformation $\gamma$ in $B^{d+1}$.

Let $T_{\mathbf{y}}^{\prime} \mathbf{x}$ be the Jacobi matrix of (A23); then

$$
T_{\mathbf{y}}^{\prime} \mathbf{x}=\frac{1-|\mathbf{y}|^{2} / R^{2}}{[\mathbf{x}, \mathbf{y}]^{2}} Q(\mathbf{y}) Q\left(\mathbf{x}-\mathbf{y}^{*}\right),
$$

with * and $Q$ defined in (A21).

The conformal change of scale $\left|T_{\mathbf{y}}^{\prime} \mathbf{x}\right|\left(\left|T_{\mathbf{y}}^{\prime} \mathbf{x}\right|^{d+1}\right.$ is the Jacobi determinant in $B^{d+1}$, $\left|T_{\mathbf{y}}^{\prime} \mathbf{x}\right|^{d}$ the Jacobi determinant of $T_{\mathrm{y}}$ acting on $\left.S_{d}\right)$ reads as

$$
\left|T_{\mathbf{y}}^{\prime} \mathbf{x}\right|=\frac{1-|\mathbf{y}|^{2} / R^{2}}{[\mathbf{x}, \mathbf{y}]^{2}}
$$

For $Q$ we have orthogonality and symmetry, $Q^{-1}=Q^{t}=Q$, and $Q(-\mathbf{x})=Q(\mathbf{x})$, and 


$$
Q(\mathrm{y}) Q\left(\mathrm{x}-\mathrm{y}^{*}\right)=Q\left(\mathrm{x}^{*}-\mathrm{y}\right) Q(\mathrm{x}) .
$$

Analogously to (A25) we have, for every Möbius transformation $\gamma$,

$$
\left|T_{\gamma \mathbf{y}}^{\prime} \gamma \mathbf{x}\right|\left|\gamma^{\prime} \mathbf{x}\right|=\left|T_{\mathbf{y}}^{\prime} \mathbf{x}\right|
$$

with $\gamma^{\prime}$ the conformal change as in (A26).

We note that the following special values of $T$ and $T^{\prime}$ all easily follow from (A23) and (A26), namely,

$$
\begin{gathered}
T_{\mathrm{y}} \mathrm{y}=0, \quad T_{\mathrm{y}}^{\prime} \mathrm{y}=\left(1-|\mathrm{y}|^{2} / R^{2}\right)^{-1} i d, \quad T_{\mathrm{y}} 0=-\mathrm{y}, \\
T_{\mathrm{y}}^{\prime} \mathbf{0}=\left(1-|\mathbf{y}|^{2} / R^{2}\right) i d, \quad T_{0} \mathbf{x}=\mathbf{x}, \quad T_{\mathrm{y}}{ }^{-1} \mathbf{x}=T_{-\mathbf{y}} \mathbf{x} .
\end{gathered}
$$

Obviously the Poisson kernel (A9) can be expressed as

$$
P(\mathbf{x}, \boldsymbol{\eta})=\left|T_{\mathbf{x}}^{\prime} \eta\right|
$$

with $\left|T_{\mathbf{x}}^{\prime} \eta\right|$ as in (A27). From (A29) we have the following transformation formulas for $P$ :

$$
P(\gamma \mathbf{x}, \gamma \boldsymbol{\eta})=P(\mathbf{x}, \boldsymbol{\eta})\left|\gamma^{\prime} \boldsymbol{\eta}\right|^{-1} \text { or } P(\gamma \mathbf{x}, \boldsymbol{\eta})=P\left(\mathbf{x}, \gamma^{-1} \eta\right)\left|\gamma^{-1^{\prime}} \eta\right|
$$

Analogous formulas hold for the $H^{d+1}$ model and $P(\mathbf{y}, t ; \xi)$ in (A2).

${ }^{1}$ R. Tomaschitz, J. Math. Phys. 32, 2571 (1991).

${ }^{2}$ R. Tomaschitz, in Chaotic Dynamics: Theory and Practice, edited by T. Bountis, NATO-ASI Series (Plenum, New York, 1992).

${ }^{3}$ R. Tomaschitz, Complex Syst. 6, 137 (1992).

${ }^{4}$ G. Hedlund, Duke Math. J. 2, 530 (1936).

5 A. Beardon, The Geometry of Discrete Groups (Springer-Verlag, Berlin, 1983).

${ }^{6}$ E. Schrödinger, Physica 6, 899 (1939).

${ }^{7}$ E. Schrödinger, Expanding Universes (Cambridge University, Cambridge, 1956).

${ }^{8}$ N. D. Birrell and D. C. W. Davies, Quantum Fields in Curved Space-Time (Cambridge University, Cambridge, 1983).

${ }_{9}^{9}$ R. Tomaschitz, Int. J. Theor. Phys. 31, 187 (1992).

${ }^{10}$ P. Scott, Bull. London Math. Soc. 15, 401 (1983).

${ }^{11}$ R. Tomaschitz, J. Math. Phys. (to be published).

${ }^{12}$ L. V. Ahlfors, "Möbius transformations in several dimensions," Lecture Notes, University of Minnesota, 1981.

${ }^{13}$ N. Mandouvalos, Proc. London Math. Soc. 57, 209 (1988). 